\title{
The role of interfacial viscoelasticity in the stabilization of an electrospun jet
}

\author{
Omri Regev $^{1}$, Steven Vandebril ${ }^{2}$, Eyal Zussman ${ }^{3}$, Christian Clasen $^{2}$ \\ ${ }^{1}$ Russel Berrie Nanotechnology Institute, Technion - Israel Institute of Technology, Haifa, Israel \\ ${ }^{2}$ Department of Chemical Engineering, Katholieke Universiteit Leuven, Leuven, Belgium \\ ${ }^{3}$ Faculty of Mechanical Engineering, Technion - Israel Institute of Technology, Haifa, Israel
}

\begin{abstract}
Stabilization of the jet is necessary for the successful fabrication of continuous fibers from solutions via electrospinning. Although intensively studied over the past decade, the mechanisms underlying jet stabilization are still not precisely understood. The traditional explanation for jet stabilization emphasizes the role of the elastic response of the polymer coil in creating a sufficiently high extensional viscosity, which prevents the breakup of the filament under extension. However, comprehensive rheological studies of bovine serum albumin (BSA) solutions that can be electrospun into continuous fibers show an absence of any significant bulk elasticity in shear and extension that would account for the stabilization of the jet. In order to explain this discrepancy, it is proposed that a complex jet structure, composed of a liquid core surrounded by a viscoelastic interface, is formed during the spinning process, where the surface viscoelasticity is responsible for the jet stabilization. These rheological properties of the surface are experimentally verified using novel interfacial rheometry. It is also shown that the surface viscoelasticity is further enhanced by varying the protein conformation (unfolding), as well as its concentration in solution.
\end{abstract}




\section{Introduction}

Electrospinning is a versatile method for the fabrication of nanofibers [1-3]. In this method a liquid, typically a polymer solution, is introduced into a strong electrostatic field, where the charged solution is drawn out into a jet. The jet then undergoes extensive stretching and thinning, with an extension rate on the order of $1000 \mathrm{~s}^{-1}$ [1], and a rapid evaporation of the solvent. Ultra-thin fibers, having diameters in the range of micrometers down to tens of nanometers, are formed on the scale of milliseconds.

Extensive research has been performed regarding why a particular solution will be spinnable or not [4-6]. It has been concluded that a spinnable solution is one where the forming jet is sufficiently stable, i.e. the filament will not break up before the final dry fiber is formed. Still, the question remains as to what the precise mechanisms are that responsible for the stabilization of an electrospun jet. McKinley's review [7] states the necessity of a sufficiently high extensional viscosity throughout the entire thinning process in order to prevent the breakup of the filament into individual droplets. However, the source of this extensional viscosity along the jet can vary [8]. It may arise from the solvent viscosity, from an elastic contribution of the dissolved polymer in the jet [9], or from an anisotropic structure which develops in the jet, giving rise to extension thickening.

For concentrated polymer solution Shenoy et al. [10] suggested a correlation between the number of entanglements per polymer chain and the electro-spinnability. According to them the stabilization of the jet requires at least one entanglement per chain in order to achieve a sufficiently high elasticity and subsequently extensional viscosity. While their mechanistic explanation is based upon the shear rheological properties of the solutions, they mention the fact that the entanglement density will also affect the extensional viscosity of these polymeric systems. Bhattercharjee et al. [11] demonstrated that further increasing the number of entanglements per polymer chain (e.g. by increasing the concentration of the solution while holding the polymer molecular weight constant) results eventually in an increased degree of extensional thinning and less stabilization of the jet as the strain rate increases due to the dramatic stretching of the jet.

However, as pointed out by $\mathrm{Yu}$ et al. [9], the required elasticity or extensional viscosity is not necessarily bound to the entanglements of a polymer solution. According to them the 
stabilization can be achieved by any sufficiently strong elastic response of the liquid, in their case even for dilute polymer solutions, where the elastic relaxation time of the system was comparable to the time scale of the filament extension.

If the extensional viscosity is not sufficiently high throughout the entire length of the electrospun jet, e.g. at locations where the strain rate is not sufficiently high to affect an elastic extension thickening, the jet will generally break apart into single droplets in a Rayleigh instability phenomenon [7, 12-14]. If the strain rate in the connecting thinning filament between the droplets becomes sufficiently high to induce an elastic response (e.g. a coil-stretch transition of the polymer chains in a dilute solutions when the time scale of extension becomes smaller than the relaxation time of the system), then the elastic stresses that result will introduce a sufficiently high extensional viscosity to stabilize the filament, thus resulting in the formation of a beads-on-string structure $[15,16]$. Decreasing the polymer concentration in a former stable electrospun jet will therefore eventually lead to a beads-on-string structure in the final fiber.

The electrospinning of solutions of naturally-occurring proteins is more challenging than of synthetic polymers, since the stability of their tertiary structure reduces their capacity to unravel in an extensional flow field, thus preventing the viscoelastic response necessary for jet stabilization. Consequently, bio-macromolecules are often blended and co-spun with synthetic polymers [17-19]. However, protein solutions also offer the possibility to change the secondary and tertiary structures of a protein with simple chemical modifications. The resulting changes in the conformation of these biomacromolecules are achieved without changing the concentration or solvent type, which is not possible for synthetic polymers. Recently, Dror et al. [20] managed to electrospin nanofibers from bovine serum albumin (BSA) by altering its electrostatic charge and reducing the intra-chain disulfide bonds, resulting in an unfolding of the protein conformation and a promoting of the formation of new inter-chain cross-links. However, no clear correlation has been established between the rheological behavior of protein solutions and the stabilization of their electrospun jets. In the present paper we therefore present a thorough rheological study of BSA solutions in different conformational state in an effort to formulate the correlation to electro-spinnability and propose a novel mechanism for the jet stabilization via the viscoelasticity of the interfacial layer of the jet. The conclusions of this study will be generally applicable to future investigations of spinnability issues regarding protein solutions (on the same 
order of molecular weight $\left(M_{w}\right)$ as BSA), and polymer solutions with similar physico-chemical properties.

\section{Materials and Methods}

\subsection{Materials and Solutions}

Various concentrations of BSA (Fraction V, MP Biomedicals) were dissolved in different mixtures of solvents, including single distilled water and 2,2,2-trifluoroethanol (TFE, ReagentPlus ${ }^{\circledR}$, Sigma-Aldrich). In certain solutions the BSA disulfide bonds were reduced by the addition of beta-mercaptoethanol ( $\beta$-ME, molecular biology grade, Merck/Calbiochem), using an amount which calculates as ten equivalences of the number of disulfide bonds within the protein molecule $(0.2 \mathrm{~g} \beta$-ME per $1 \mathrm{~g}$ of BSA $)$, see Table 1 .

\begin{tabular}{|c|c|c|c|}
\hline Solution & Composition & As-spun morphology & $\mathrm{pH}$ \\
\hline$\# 1$ & $10 \%(w / w) \mathrm{BSA}$ in $\mathrm{H}_{2} \mathrm{O}$ & Drops & 7.00 \\
\hline$\# 2$ & $10 \%(\mathrm{w} / \mathrm{w}) \mathrm{BSA}$ in TFE: $\mathrm{H}_{2} \mathrm{O} 9: 1(\mathrm{w} / \mathrm{w})$ & Fibers fragments & 6.90 \\
\hline$\# 2 \mathrm{a}$ & $20 \%(w / w)$ BSA in TFE: $\mathrm{H}_{2} \mathrm{O} 9: 1(\mathrm{w} / \mathrm{w})$ & Fibers fragments & 6.98 \\
\hline$\# 3$ & $10 \%(\mathrm{w} / \mathrm{w}) \mathrm{BSA}$ in TFE: $\mathrm{H}_{2} \mathrm{O} 9: 1(\mathrm{w} / \mathrm{w})$ and $10 \mathrm{eq} \beta-\mathrm{ME}$ & Long fibers & 6.90 \\
\hline$\# 3 \mathrm{a}$ & $20 \%(\mathrm{w} / \mathrm{w}) \mathrm{BSA}$ in TFE: $\mathrm{H}_{2} \mathrm{O} 9: 1(\mathrm{w} / \mathrm{w})$ and $10 \mathrm{eq} \beta-\mathrm{ME}$ & No jet formation & - \\
\hline
\end{tabular}

Table 1 - List of tested albumin solutions

\subsection{Electrospinning}

Electrospinning was conducted at room temperature, using a syringe pump (Harvard Apparatus), a 23 Gauge needle (inner diameter $\sim 0.37 \mathrm{~mm}$ ), and a custom-built high-voltage (30 $\mathrm{kV}$ max.) DC supply, while fibers were collected on various collectors (small and large plates, rotating disk, as illustrated in Theron et al. [21]). For each solution, a wide range of values for the electrospinning parameters was used, including applied voltage $(3-30 \mathrm{kV})$, solution flow rate $(0.1-5.0 \mathrm{ml} / \mathrm{hr})$, and needle-to-collector distance $(5-20 \mathrm{~cm})$. The collected structures were characterized using an optical microscope (Olympus BX51) and a high-resolution scanning electron microscope (HR-SEM, Carl Zeiss LEO 982).

\subsection{Shear Rheometry}

Shear measurements were conducted using a strain controlled rotational rheometer (ARES-LS, Rheometric Scientific) with a cone-and-plate geometry $(50 \mathrm{~mm}, 0.02 \mathrm{rad})$ and a 
stress controlled rotational rheometer (Physica MCR 501, Anton Paar) with a cone-and-plate geometry $\left(50 \mathrm{~mm}, 1^{\circ}\right.$ angle). In order to ensure measurement of the actual bulk properties, evaporation of the solvents was prevented using the solvent traps provided with the rheometers. After conducting a stress/strain sweep test to determine the linear viscoelastic regime, a frequency sweep test was performed in order to check for the existence of elasticity in the solutions. Creep (transient) and flow curve (stress/rate step) tests were then carried out in order to investigate the behavior of the solutions under steady rotation.

\subsection{Extensional Rheometry}

Extensional measurements were conducted using two testing devices. The first, a custombuilt extensional rheometer [22], captures the diameter evolution of a liquid filament following a fast initial step strain, using a high speed camera (MotionScope8000, $500 \mathrm{fps}, 1 / 1000 \mathrm{sec}$ ). The images are then processed to obtain the full profile of the thinning filament. The second tester is a capillary breakup extensional rheometer (CaBER, Thermo Fisher Scientific), which uses a laser micrometer to directly measure the evolution of the mid-filament diameter over time [7]. The CaBER allows for a very precise determination of small filament radii $(>10 \mu \mathrm{m})$, while the custom-built rheometer provides a full filament profile analysis. This analysis permits a further interpretation of the results from the CaBER, which is essential for filaments that break up in a non-uniform pattern.

The extensional viscosity at a constant strain rate was determined using a custom-built filament stretching rheometer (fiser) described elsewhere [23, 24].

\subsection{Interfacial Rheometry}

Interfacial rheological properties were determined using a novel double-wall ring geometry (details described elsewhere $[25,26]$ ) mounted to a rotational rheometer (AR-G2, TA Instruments). The teflon cup holding the sample was filled to approximately $1 \mathrm{~mm}$ (sample volume $\sim 18 \mathrm{ml}$ ). The ring was positioned at the interface visually, and the sample was deliberately kept in direct contact with the ambient atmosphere in order to allow evaporation across the sample surface. The interfaces were then probed by means of a small-amplitude oscillatory shear (SAOS) experiment, at a frequency of $0.1 \mathrm{~Hz}$ and a strain amplitude of $1 \%$. The sample height was checked every $90 \mathrm{~min}$, and the ring position adjusted if necessary. 


\subsection{Surface Pressure}

Surface pressure measurements were conducted with a Wilhelmy balance (KSV Instruments). Initially, the balance contained $30 \mathrm{ml} \mathrm{MilliQ}$ water, into which a sample $(10 \mu \mathrm{l})$ of a protein solution was injected. The sample was injected directly into the bulk, avoiding the penetration of the interface.

\section{Results and Discussion}

\subsection{Spinnability}

The electrospun protein solutions are classified as one of three representative structures that are shown in Fig. 1. Electrospinning of solution \#1 (the native protein) resulted in drops (see Fig. 1a), similar to the product of an electro-spraying process. Electrospinning of solution \#3 (the unfolded protein) resulted in continuous fibers (see Fig. 1c), whereas the electrospinning of solution \#2 (the partially-unfolded protein) produced an intermediate structure of fiber fragments with non-uniform diameter, similar to beads-on-string structures (see Fig. 1b). Electrospinning of the higher concentrated solution \#2a (of the partially-unfolded protein) resulted in a similar beads-on-string fiber fragments. The continuous fibers of solution \#3 have a smooth surface and a cylindrical- or ribbon-like morphology (Fig. 1c). The ribbon shape is most likely a result of the collapsing of the fibers due to entrapped solvents [27]. The diameter of the cylindrical fibers is in the range of 500-1000 $\mathrm{nm}$. Electrospinning of the higher concentrated solution \#3a was not possible due to the rapid drying of this solution which prevented the formation of a jet at the needle tip. 


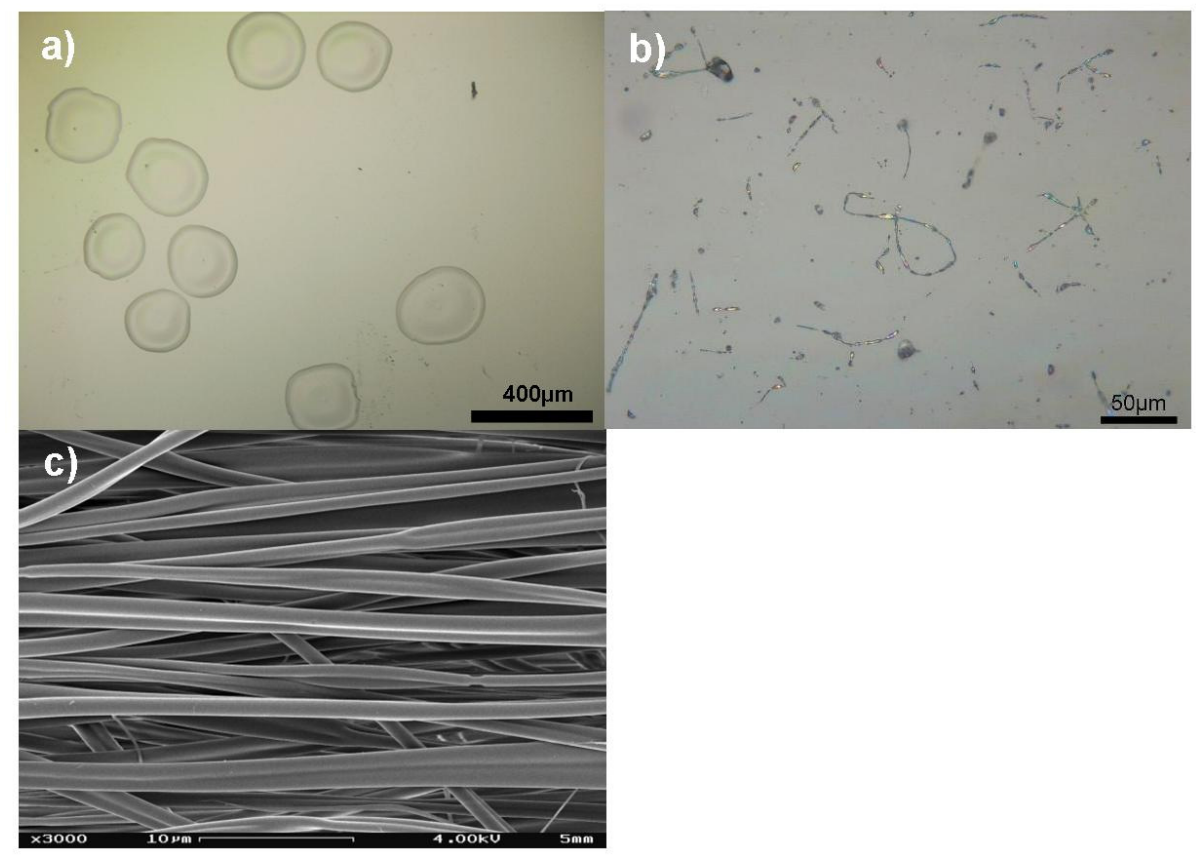

Figure 1: Electrospinning results. Optical microscope images of (a) Drops from solution \#1, (b) Fragments of fibers from solution \#2; and (c) SEM image of as-spun fibers from solution \#3.

A rheological study was performed on each of the solutions shown in Fig. 1 and Table 1 in order to find a correlation between the viscoelastic properties of these protein systems and the stabilization of their electrospun jets. Analyzing the electrospinning process solely on the basis of the viscoelastic properties and rheological steady state values is a considerable simplification, since electrospinning is a transient process with an evolving extensional viscosity, which is affected by additional effects such as solvent evaporation and non-homogeneous change of the polymer concentration and solution conductivity. Furthermore, the whipping instability that the filament experiences during the spinning process leads to flow conditions that are beyond the shear and extension rate capabilities of current rheological experiments. Still, the approach of using only the abstract rheological material properties provides a versatile method for the comparison of the three protein solution systems, in order to determine distinctions that can be used to qualitatively explain the electro-spinnability of protein solutions.

\subsection{Shear Rheology}

Figures 2 and 3 show the dynamic and steady-state viscoelastic properties of the investigated solutions. 


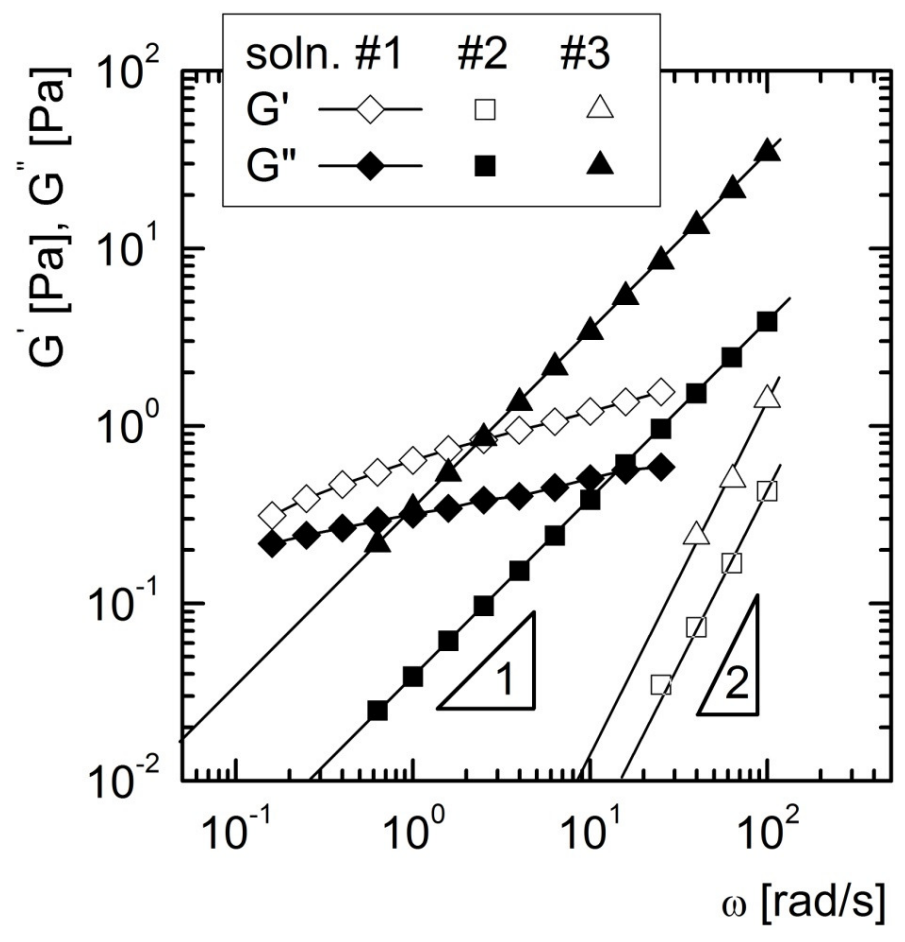

Figure 2: Storage modulus $G^{\prime}(\omega)$ and loss modulus, $G^{\prime \prime}(\omega)$ as a function of applied frequency, as determined in a small-amplitude oscillatory shear (SAOS) experiment. The oscillation amplitude was held within the linear viscoelastic regime, as follows: solution \#1 (measured with Physica rheometer) at a constant stress amplitude of $\sigma=0.01 \mathrm{~Pa}$; for solutions \#2 and \#3 (measured with ARES rheometer), at a constant strain amplitude of $\gamma=20 \%$. Measurements of solution \#3 were performed 6 hours after the addition of $\beta$-ME. 


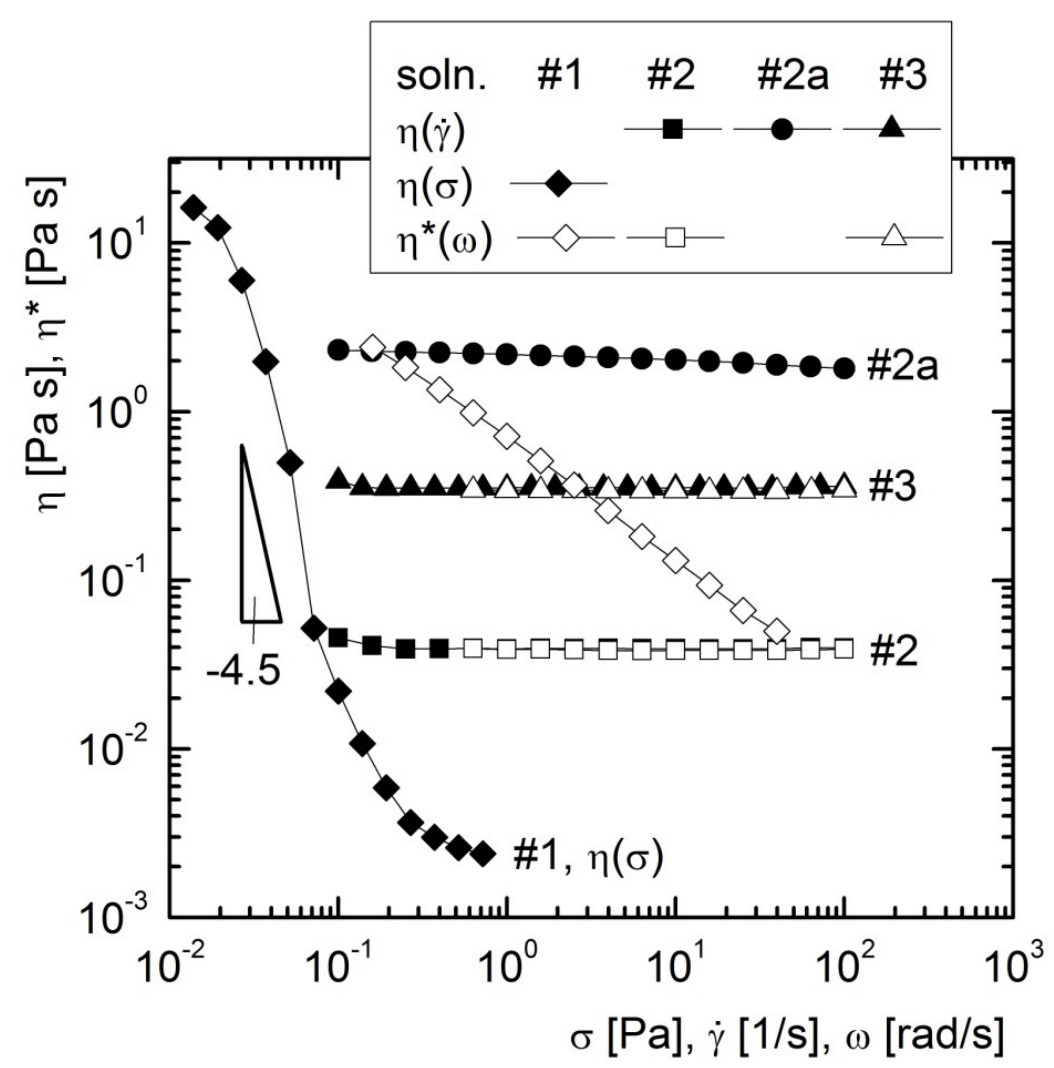

Figure 3: Shear viscosity $\eta$ and complex viscosity $\eta^{*}$ as a function of shear stress $\sigma$, shear rate $\dot{\gamma}$, and angular frequency $\omega$, determined for various BSA solution samples. The viscosity as a function of the shear stress $\eta(\sigma)$ for solution \#1 was determined using a stress-controlled rheometer (for each point measuring time is $5 \mathrm{~min}$ ). The viscosity as a function of the shear rate $\eta(\dot{\gamma})$ for solutions \#2, \#2a, and $\# 3$, was determined using a rate-controlled ARES rheometer (for each point the waiting time to steady-state and measuring time are $20 \mathrm{~s}$ and $2 \mathrm{~s}$, respectively). The complex viscosity $\eta^{*}$ is calculated from the respective moduli shown in Fig. 2. All measurements of solution \#3 were conducted $6 \mathrm{hr}$ after the addition of $\beta$-ME.

The globular albumin solution \#1 exhibits a yield stress due to the formation of a colloidal crystal, in which the compact spheres of the native BSA are arranged in a lattice due to inter-molecular non-covalent interactions [28]. This is clearly seen in Fig. 2, where a solid-like behavior is indicated by an elastic storage modulus $G$ ' that is nearly independent of the applied frequency $\omega$. A yielding of this internal microstructure is observed in Fig. 3 by the sharp drop in viscosity of solution \#1 of 4 decades over a range of shear stresses $\sigma=0.02-0.2 \mathrm{~Pa}$, resulting in the onset of flow. A rough estimation of the capillary pressure $p=2 \gamma / D$ that acts on a liquid column of solution \#1 with diameter $D$ and surface pressure $\gamma$, implies that, for diameter below $D$ $\sim 0.4 \mathrm{~mm}$, the yield stress level has already been reached. The yield stress of solution \#1 
therefore does not play a role in the stabilization of an electrospun jet, and the effective shear viscosity during the spinning process is close to that of the solvent (water), i.e. on the order of 2 mPa s.

In solution \#2, the protein's native structure is partially altered due to the good solvating properties of the TFE, and the protein chains are stabilized in a so-called partially-unfolded state, as described elsewhere [29-31]. Additionally, the TFE prevents inter-molecular interactions between the partially-unfolded protein coils, preventing the formation of an organized lattice in this solution and resulting in a Newtonian flow behavior. The steady-state shear viscosity in Fig. 3 has a constant value $\eta=0.04 \mathrm{~Pa} \mathrm{~s}$, which is 20 times larger than that for solution \#1 (pure BSA/water system), due to the increased volume which the partially-unfolded protein structure occupies. The oscillatory experiment data in Fig. 2 show that an elastic storage modulus $G^{\prime}$ is still detectable at higher values of the frequency $\omega$. The observed scaling of G' $\sim \omega^{2}$ and $G^{\prime \prime} \sim \omega$ indicate that this range of frequencies is the terminal regime of linear viscoelasticity, thus allowing for an estimation of a relaxation time of the solution

$$
\tau=\lim _{\omega \rightarrow 0} \frac{G^{\prime}}{G^{\prime \prime} \omega},
$$

which calculates to $\tau=5 \times 10^{-4} \mathrm{~s}$.

The low elasticity of solution \#2 is attributed to a slight onset of entanglements of segments of the partially-unfolded protein coils. Increasing the protein concentration to $20 \%$, the viscosity increases by a factor of 50 , to $\eta=2 \mathrm{~Pa}$ s (shown as solution \#2a in Fig. 3). However, the more concentrated solution still exhibits Newtonian behavior with a negligible elastic modulus (data not shown).

The addition of $\beta$-ME to solution \#3 reduces the intra-molecular disulfide bonds of the protein. As demonstrated by Dror et al. [20], the backbone of the protein chain remains intact, and there is no formation of new inter-molecular disulfide bonds as long as the protein's environment contains $\beta$-ME. Solution \#3 consists, in principal, of a linear polymer with $M_{w} \sim 66$ $\mathrm{g} / \mathrm{mol}$. The complete unfolding of the protein in solution \#3, due to the combined effects of $\beta$ $\mathrm{ME}$ and the solvent (TFE), leads to a further increase in the volume occupied by the protein chains. As a result, the viscosity increased to $\eta=0.35 \mathrm{~Pa} \mathrm{~s}$ (as shown in Fig. 3). The solution behaves still as a Newtonian fluid, and also the dynamic results of Fig. 2 show that even for the free protein coils there is no significant increase in the storage modulus $G^{\prime}$ and hence elasticity 
at this concentration, and therefore no indication of pronounced entanglements. The relaxation time which can be estimated from the moduli of Fig. 2 is $\tau=\lim _{\omega \rightarrow 0}\left(G^{\prime} / G^{\prime \prime} \omega\right)=4 \times 10^{-4} \mathrm{~s}$, which is quite similar to that of solution \#2. Solution \#3 shows its rapid increase in viscosity already within minutes after the addition of $\beta-\mathrm{ME}$ in comparison to solution \#2. However, the chemical reactions of breaking of disulfide bonds continue to take place with the passage of time. This continuous increase in viscosity, as shown for the complex viscosity $\eta *$ in Fig. 4 (open symbols), is measured at successive time intervals, following the addition of $\beta$-ME (the data for solution \#3 in Fig. 3 actually represent the status 6 hours after the addition of $\beta$-ME). While the significant viscosity changes occur within the first 6 hours following the addition of $\beta$-ME, the process is not fully completed even after 22 hours. It should be noted that the time period of 6 hours is more than is necessary to in order to successfully electrospin solution \#3 into long fibers.

The stabilization of a jet in the electrospinning process can for certain cases already be explained outgoing from the shear rheological material functions determined above. For example, Kowalewski et al. [32] demonstrated for the case of glycerol a stabilization purely by the viscous forces of the Newtonian liquid. However, this viscosity-controlled stabilization is possible only to stretch ratios $\varepsilon$ on the order of $\mathrm{O}(10)$. Beyond that the onset of another stabilization mechanism is required to prevent the disintegration of the stretched viscous jet into droplets via the development of a Rayleigh instability.

A stabilization of the filaments in the present investigation solely due to the viscosity can also be ruled out by comparing the viscosities of unfolded solution \#3 and partially-unfolded but higher concentrated solution \#2a. In spite of a viscosity level higher than that of solution \#3 (see Fig. 3), solution \#2a (no $\beta$-ME added) remains only partially-spinnable and breaks ultimately into small fiber fragments.

Also an elastic stabilization and contribution to the spinnability of solution \#3 due to a sufficient number of entanglements per chain as discussed by Shenoy et al. [10] can be ruled out for the present investigation. Although the unfolding of the protein due to the addition of $\beta$-ME in solution \#3 increases the polymer coil volume compared to solution \#2 (indicated by the increase in shear viscosity), the low elastic response of the solutions observed in Fig. 2 indicates that solution \#3 is not significantly entangled. 


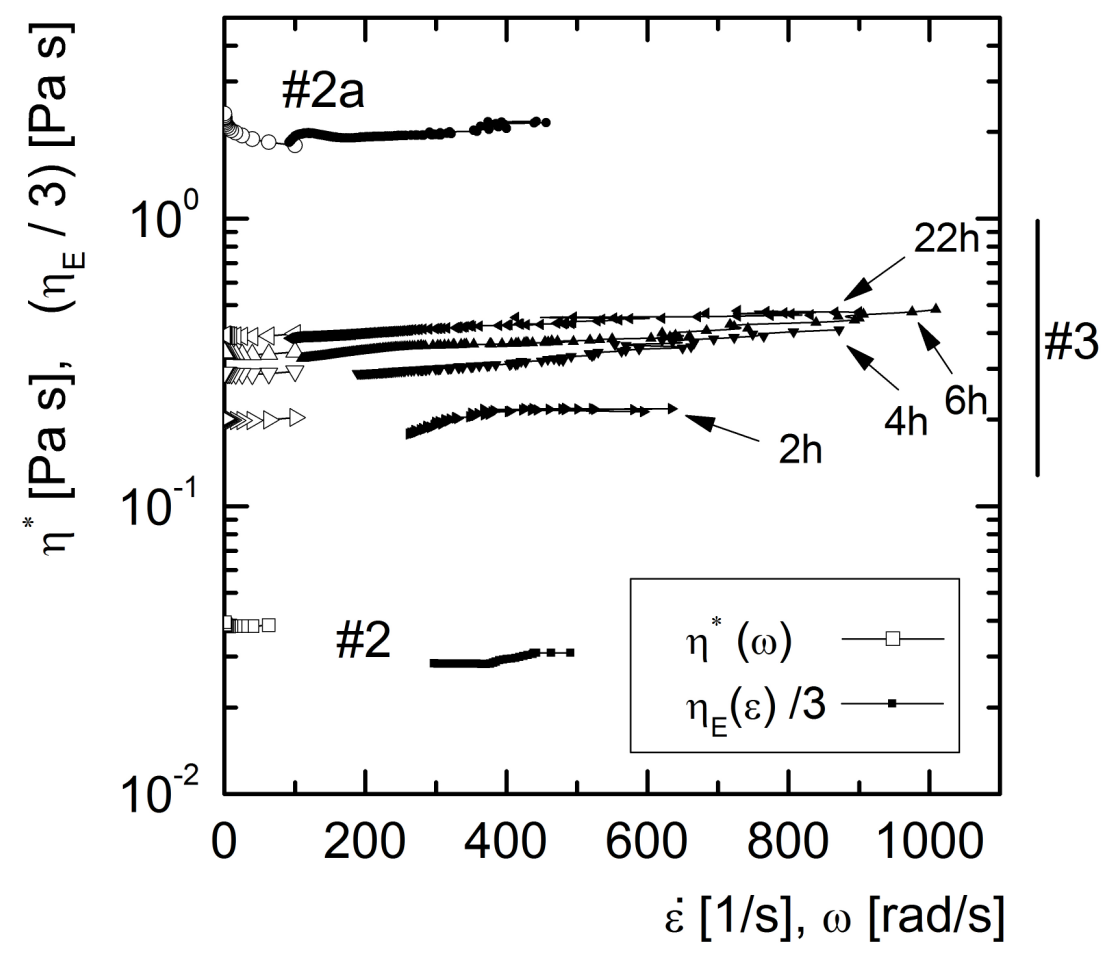

Figure 4: Comparison of the complex viscosity $\eta^{*}$ (as a function of the frequency, with a constant strain amplitude of $20 \%$ ) and the apparent extensional viscosity $\eta_{\mathrm{E}}$ (as a function of the strain rate $\dot{\varepsilon}$, as determined using the CaBER) for solutions \#2 and \#3. The measurements were carried out at 2, 4,6, and $22 \mathrm{hr}$ following the addition of $\beta$-ME.

\subsection{Extensional Rheology}

It is important to recognize that the steady shear flow experiments in the previous section probed only the linear response of the dissolved polymers and do not necessarily reflect the full flow profile of a filament undergoing the electrospinning process. The strong extensional component is capable of inducing a non-linear response of the polymer in solution which can be quite different from the shear properties $[15,33,34]$. As for example shown by $\mathrm{Yu}$ et al. [9], the unraveling of the polymer chains in the strong extensional flow field lead in their case to a stabilization of the liquid filament that was caused by entropy-elastic stresses of the stretched polymer coils. Therefore, the following experiments are conducted to determine the viscoelastic response in an extensional flow which more closely resembles that which the filament undergoes during electrospinning. 

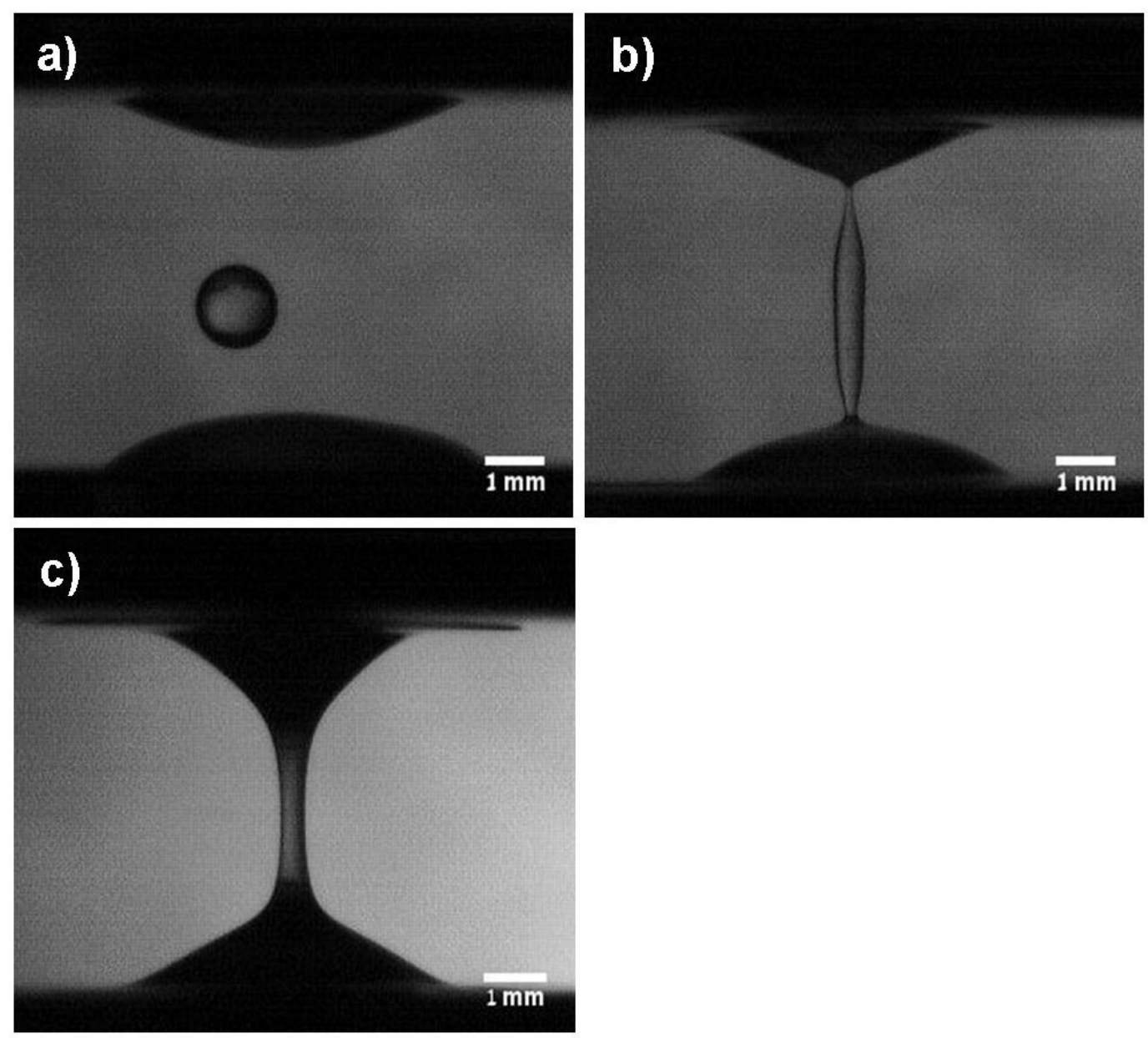

Figure 5: High-speed images taken immediately following the separation of the endplates in the extensional rheometer trials. (a) solution \#1, (b) solution \#2, and (c) solution \#3. The endplates separation velocity is $\sim 22 \mathrm{~cm} / \mathrm{s}$

The basic differences in the extensional flow behavior of the three protein solutions are seen in Fig. 5. These images, captured with a high-speed camera, show the results of the capillary thinning and breakup of the solutions during and after a rapid extension, as performed in a capillary-breakup experiment [22]. Solution \#1 and \#2 form no stable filaments and break already during or shortly after the initial rapid extension into droplets (Fig. 5a, b). In contrast, solution \#3 forms stable filaments that decay linearly in time, as can be seen in Fig. 6a that shows the filament diameter evolution of the image sequences of Fig. 5 as a function of time. 

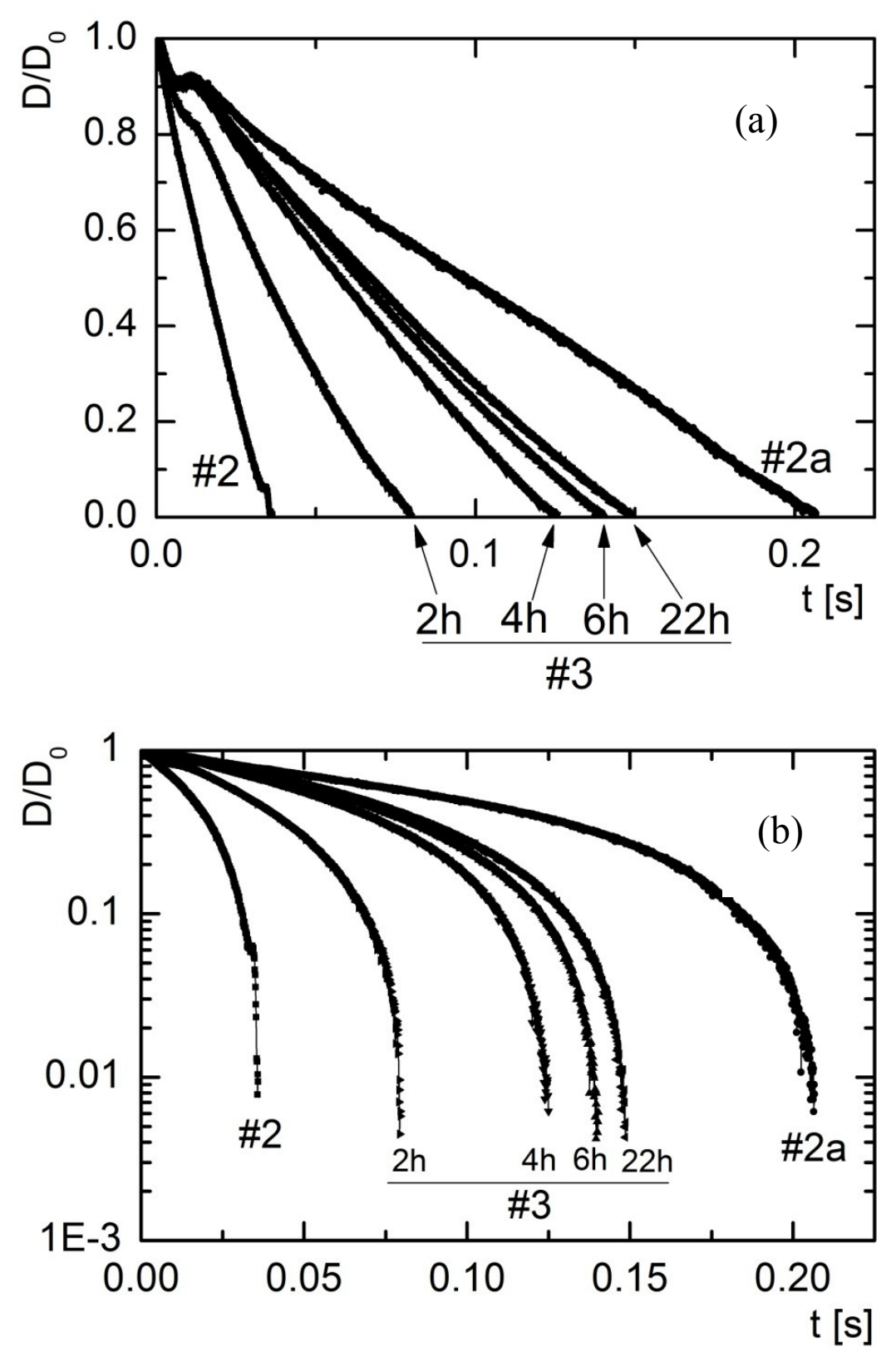

Figure 6: Diameter $D$ normalized with the initial filament diameter $D_{0}=1 \mathrm{~mm}$ as a function of time for the different protein solutions. The data for solution \#2 and \#3(6h) refer to the image sequences in figure $5 \mathrm{~b}$ and $\mathrm{c}$, respectively.

This behavior could already be expected from the shear viscosities of solutions, since the Ohnesorge number $O h=\eta / \sqrt{D_{0} \rho \gamma / 2}$ (which compares the timescales of viscous- and inertiacontrolled breakup) allows to estimate if a stable filament can be observed [35]. Using the relevant values for shear viscosity $\eta$ from Fig. 3, the initial filament radius $D_{0}$ (observed 
experimentally from the high-speed images), fluid density $\rho$ (determined experimentally from solution mass and volume), and surface tension $\gamma$, the Ohnesorge numbers are calculated for solutions \#1, \#2 and \#3 to be $0.0041,0.15$ and 1.35, respectively. Since the values for solutions $\# 1$ and \#2 are below unity, viscous forces are insufficient to stabilize the filament, and the jet will break up into droplets due to the development of a Rayleigh instability [12, 14]. The yield stress of solution \#1 is not relevant, since the capillary pressure in the initial filament is $2 \gamma / D_{0} \approx 20 \mathrm{~Pa}$ which is about an order of magnitude greater than the upper limit for the range of yield stresses (see Fig. 3).

In comparison to the electrospinning process where the extension of the filament is driven by combined electrical/surface tension force, the thinning experiments in Fig. 5 and 6 are solely surface tension driven. Still they allow estimating the transient viscoelastic response of the sample for increasing extension rates as experienced in the collapsing filaments. A transient apparent extensional viscosity $\eta_{E, a p p}=\sigma_{11} / \dot{\varepsilon}$ can be determined by the general definition of extension rate, via the diameter evolution

$$
\dot{\varepsilon}=-\frac{1}{D} \frac{d D}{d t}
$$

and assuming the stress in the liquid is arising from surface tension $\sigma_{11}=2 \gamma / D[36,37]$ we get

$$
\eta_{E, a p p}=-\frac{\gamma}{\frac{d D}{d t}}
$$

The apparent extensional viscosity as a function of the extension rate $\dot{\varepsilon}$ is given in Fig. 4 for solutions \#2 and \#3. Since the capillary pressure in the filaments increases as the radius decreases, the extension rate continuously increases throughout the thinning experiment. As already indicated by the quasi linear thinning of the filament diameter with time in Fig 6a, $\eta_{E, a p p}$ is constant over the range of observable filament diameters and extension rates up to $\dot{\varepsilon}=1000 \mathrm{~s}^{-}$ 1 . The apparent extensional viscosity of solution \#3 increases with time after the addition of $\beta$ ME, similar to the shear viscosity (see Fig. 4), and indeed the observed shear viscosities (also shown in Fig. 4 as open symbols) and the apparent extensional viscosities follow Trouton's ratio for simple Newtonian fluids, i.e. $\eta=\eta_{E} / 3$. Trouton's ratio holds also for higher protein 
concentrations, as can be seen for the extensional viscosity of solution \#2a (also shown in Fig. 4) for which the BSA concentration was increased to $20 \%$.

In summary, the unfolding of the protein structure in solution \#3 does not lead to an increased extensional viscosity in comparison to the shear viscosity as observed for example by $\mathrm{Yu}$ et al. [9]. A stabilization of the filament in Fig. 6 due to a transient increase of the elastic stresses of the unraveling polymer chain in the extensional flow field is not observed for the accessible range of extension rates in Fig. 4, which is on the order of that reported for the electrospinning process, i.e. $\mathrm{O}\left(10^{3} \mathrm{~s}^{-1}\right)$ [1]. This is actually not unexpected, as the elastic response of unraveling polymers will only be triggered at a critical extension rate $\dot{\varepsilon}$ (and independently of the stresses that actually cause the thinning of the filament, e.g. the combined electrical/surface tension in the electrospinning process or only surface tension in the CaBER experiments of Fig. 4). This critical extension rate $\dot{\varepsilon}$ can be estimated from the relaxation times $\tau$ of eq. (1) [9]. A coil-stretch transition of the polymer chain which leads to a transient extension thickening, and therefore a stabilization of the filament, is expected to occur when the critical Weissenberg number $W i=\tau \dot{\varepsilon} \geq 0.5$ [38]. Taking into account the calculated relaxation times $\tau$ for solutions \#2 and \#3 (see section 3.2), an onset of elastic effects is expected to occur not below extension rates on the order of $\mathrm{O}\left(10^{4} \mathrm{~s}^{-1}\right)$ and the bulk protein solutions are thus expected to exhibit the Newtonian behavior in the extension rate regimes accessed in Fig. 5 and 6 for the capillary thinning experiments.

Nonetheless an elastic stabilization of the electrospun jet of BSA cannot be completely ruled out by these investigations. We are lacking a direct experimental prove that the extension rates encountered in the electrospinning of the BSA solutions are within the reasonable estimate of $\mathrm{O}\left(10^{3} \mathrm{~s}^{-1}\right)$. Therefore there is a chance that for BSA solutions the extension rates are actually a decade higher and reach an order of magnitude that can trigger the onset of an increasing extensional viscosity. However, in the following section we present a novel mechanism of filament stabilization via interfacial effects that, even for the presence of a viscoelastic contribution of the bulk, cannot be neglected and will substantially contribute to the electrospinnability of solution \#3 into long fibers following the addition of $\beta$-ME.. 


\subsection{Interfacial effects in thinning filaments}

The importance of interfacial or surface layers during the thinning process of a liquid filament becomes obvious when considering the increase of surface area-to-volume ratio $A / V$ of the electrospun jet. The ratio of the area of the surrounding thin outer layer to the volume of an incompressible liquid core increases with the inverse of the diameter, namely $A / V=4 D^{-1}$. The jet diameter decreases over the course of the spinning process roughly from $1000 \mu \mathrm{m}$ to $0.1 \mu \mathrm{m}$ and thus the surface area-to-volume ratio is increased by four orders of magnitude. However, interfacial effects will only play a role for the stabilization of electrospun filaments when the axial forces $F_{\sigma}=(\pi D) \sigma_{\sigma}$ acting in the interface become of order of the elongational forces $F_{E}=\left(\pi D^{2} / 4\right) \sigma_{E}$ in the bulk liquid core of the filament. Here $\sigma_{\sigma}=\eta_{\sigma} \dot{\varepsilon}_{\sigma}$ is the line tension with which the surface is resisting the stretching deformation and $\sigma_{E}=\eta_{E} \dot{\varepsilon}$ is the normal stress in the bulk of the liquid filament. This force balance is described in a non-dimensional form by a Boussinesq number $[39,40]$

$$
B o=\frac{F_{\sigma}}{F_{E}}=\frac{4 \sigma_{\sigma}}{\sigma_{E} D},
$$

It is important to note that the surface area $A$ of an incompressible thinning liquid filament is undergoing a dilatational deformation $\varepsilon_{\sigma}=d A / A$ with an increasing surface area with time. The dilatational deformation rate is then

$$
\dot{\varepsilon}_{\sigma}=\frac{1}{A} \frac{d A}{d t} .
$$

Since the deformation rate of the interface $\dot{\varepsilon}_{\sigma}$ and the elongation rate of the bulk liquid $\dot{\varepsilon}$ are related due to volume conservation via

$$
2 \dot{\varepsilon}_{\sigma}=\dot{\varepsilon},
$$

this Boussinesq number can also be expressed with the respective viscosities

$$
B o=\frac{2 \eta_{\sigma}}{\eta_{E} D}
$$

The balance of these forces in a Boussinesq number of $B o=\mathrm{O}(1)$ results in a critical diameter that is determined by the ratio of the interfacial and bulk stresses or viscosities:

$$
D_{c r i t, B o}=\frac{4 \sigma_{\sigma}}{\sigma_{E}}=\frac{2 \eta_{\sigma}}{\eta_{E}},
$$


Only when the filament diameter drops below this critical value, will interfacial properties as surface viscoelasticity provide enough force in comparison to the bulk fluid to be accountable for stabilizing the filament.

Several attempts to determine interfacial rheological properties of protein solutions have been reported in the literature [41-44]. However, only recently have methods been introduced that provide the necessary sensitivity to reliably determine the viscoelastic interfacial properties of protein solutions under shear $[25,26]$. Unfortunately, there is so far no technique that could probe the actual dilatational material properties of the interface at the relevant dilatation rates of order $10^{3} \mathrm{~s}^{-1}$ and higher, in order to determine the absolute value of the stabilizing tension in the surface layer. Still, although the material properties in shear and dilatation cannot directly be compared, the shear properties of the interface allow an order of magnitude estimation of the interfacial dilational stress $\sigma_{\sigma}$ that enters the Boussinesq number in eq. (4).

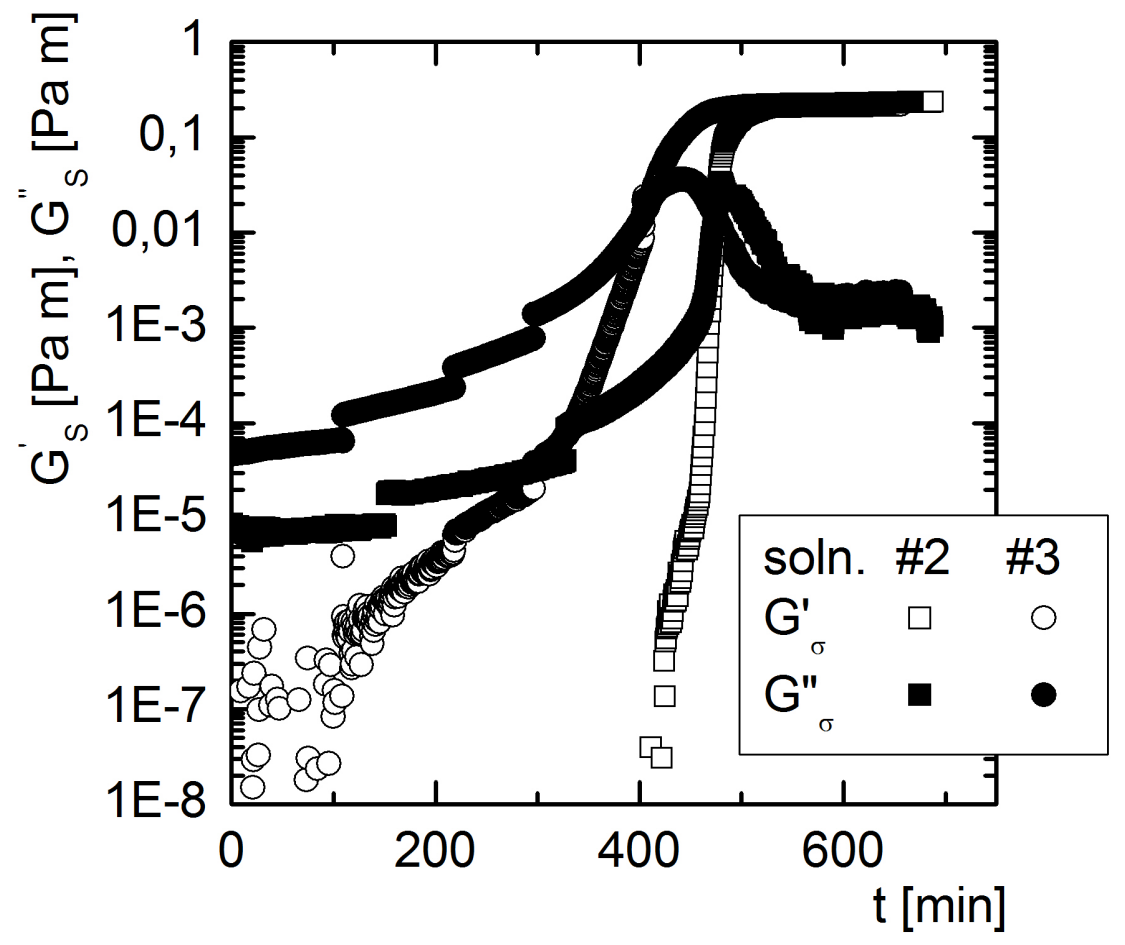

Figure 7: Interfacial storage modulus $G_{S}^{\prime}$, and loss modulus $G_{S}^{\prime \prime}$, as functions of time, as determined with a small amplitude oscillatory shear (SAOS) experiment (at a constant frequency of 0.1 $\mathrm{Hz}$ and strain amplitude of $1 \%$ ). The position of the ring geometry towards the retracting solution surface was adjusted in discontinuous steps, resulting in the step-like trends observed for the moduli. The experiments for solution \#3 were conducted 6 hours following the addition of $\beta$-ME. 
In Figure 7, the temporal evolution of the interfacial storage and loss moduli $G_{S}^{\prime}$ and $G_{S}^{\prime \prime}$ of the partially-spinnable solution (solution \#2) and the spinnable solution (solution \#3) is shown. These interfacial shear moduli were determined in a novel double-wall ring interfacial rheometer (with an oscillatory experiment similar to that of Fig. 2, but at a single frequency of $0.1 \mathrm{~Hz}$ ), which tracked the change of the interfacial properties of a static interface over time. The evolution of the interfacial shear moduli is due to a further reorganization of the conformation of the protein molecules and the solvent transfer across the interface. However, important for the electrospinning process it is the interfacial state at $t=0$ in Fig. 7 , as this represents the initial steady state of the newly created surface after the initial rapid unfolding and adsorption of protein molecules. The interfacial loss modulus $G_{S}^{\prime \prime}$ which stands for the viscous properties of the interface is nearly a decade larger for solution \#3 than for solution \#2 (and this viscosity is independent of the viscosity of the bulk). Furthermore, solution \#3 develops an elastic interfacial modulus $G_{S}^{\prime}$ immediately upon the exposure of the newly formed interface, while solution \#2 shows no detectable initial elasticity.

The origin of the differences in the interfacial shear rheological response is likely to be the higher degree of protein unfolding in solution \#3, which allows for the formation of a higherordered structure at the interface, since the hydrophobic and hydrophilic regions can more easily interact, orient, and align. Furthermore, due to evaporation which occurs at the interface, a concentration gradient of the protein molecules is formed, yielding a higher concentration at the interface, which is likely to cause an increase of interfacial viscoelasticity. The rates of this mass transfer of solvent molecules at the interface are then strongly affected by the denaturation and unfolding state of the protein itself. When more hydrophobic regions will be exposed to the air, the solvent (TFE) molecules are more weakly bound to the unfolded protein molecules, thus increasing the mass transfer of solvent molecules across the interface. This hypothesis is supported by empirical observations of the solution/air interfaces of higher concentrations of solution \#3 and \#2. Measurements of solution \#3a (20\% (w/w) BSA with added $\beta$-ME) on the CaBER were not possible since rapid solvent evaporation led to a nearly-instantaneous solidification of the forming filaments. This phenomenon was not observed for the nondenaturated solution $\# 2 \mathrm{a}(20 \%$ (w/w) BSA, with no added $\beta-\mathrm{ME})$, in which filaments formed and then broke up in the CaBER experiment (Fig. 6), with no substantial loss of solvent observed. 
Regardless of these (speculative) actual sources of the strongly increased interfacial viscoelastic response of solution \#3, we can now use the experimentally observed moduli from Fig. 7 , in order to estimate the axial forces in the interface $F_{\sigma}$, for elongation rates and filament diameters similar to those of the electrospinning process. With the high viscous modulus $G_{S}^{\prime \prime}$ at $\mathrm{t}$ $=0$ (Fig. 7) we can assume the dynamic interfacial viscosity $\eta_{S}^{\prime}=G_{S}^{\prime \prime} / \omega=6.0 \times 10^{-5} \mathrm{~Pa} \cdot \mathrm{s} \mathrm{m}$ to be the dominant contribution to the interfacial shear viscosity and use this to estimate the material function for the dilational deformations of the interface, hence $\eta_{S}^{\prime}=\eta_{\sigma}$. Using this value in eq. (8) as well as an extensional viscosity of $\eta_{E}=1.5 \mathrm{~Pa}$ s from Fig. 4, we obtain a critical diameter for the filament of $D_{\text {crit }}=80 \mu \mathrm{m}$, below which the Boussinesq number of eq. (7) becomes larger then unity and interfacial forces will start to dominate over the bulk solution forces. The interfacial viscoelastic properties of protein solutions can therefore provide enough stabilizing axial force in the diameter regime below $50 \mu \mathrm{m}$ (which was the lower resolution limit of the extensional experiments (see Fig. 6b)) and will contribute significantly to the stabilization of the jet to the final stages of thinning in the electrospinning process.

This value of $D_{\text {crit }}=80 \mu \mathrm{m}$ is clearly order of magnitude estimation for several reasons

- The experiment in Fig. 7 probes only the linear response of the interface. The thinning dynamics of the filament during the electrospinning process is likely to induce also non-linear deformation and strain hardening of the interface [45, 46]. The dynamic interfacial viscosity $\eta_{S}^{\prime}$ would then be lower than the actual interfacial viscosity $\eta_{\sigma}$. Furthermore, in this experiment only the response to shear deformation is probed, while the interface in the spinning process experiences a combined dilatational/elongational deformation.

- The initial steady state value of the dynamic interfacial viscosity $\eta_{S}^{\prime}$ obtained from Fig. 7 at $\mathrm{t}=0 \mathrm{~s}$ is likely to be overestimated, as the loading time in this experiment reaches diffusion timescales and the formation of multiple protein layers at the interface could have already started up [47, 48]. In addition, the timescale of entanglement formation between the adsorbed molecules at the interface is not yet investigated. If a possible interfacial entanglement formation is faster than the loading time, the dynamic interfacial viscosity $\eta_{S}^{\prime}$ will be higher than the actual interfacial 
viscosity $\eta_{\sigma}$ during the thinning process of the filament. The time evolution of the modulus indicates that after the starting point of experiment the progress is slow and over the course of minutes, however this gives no information on the initial evolution, in timescale of seconds.

In particular the last point on the relevant timescales requires an in-depth discussion. Although we have just shown that the steady state interfacial material properties reach order of magnitude that are sufficient to stabilize a filament, the dilatational deformation in the thinning filament is continuously creating new surface that still has to acquire steady state properties. The following section is therefore investigating how fast a newly created interface of solution \#2 and \#3 will reach this steady state.

\subsection{Adsorption kinetics}

The line tension $\sigma_{\sigma}$ in eq. (8), and therefore the resisting force $F_{\sigma}$ acting in the interface against the dilatational deformation depend strongly on the population and conformation of the proteins at the interface. Due to continuous creation of new surface in the dilatational deformation, the apparent interfacial viscosity $\eta_{\sigma}=\sigma_{\sigma} / \dot{\varepsilon}_{\sigma}$ (and therefore the stabilizing effect of the surface on the overall filament) is strongly depending on the absorption kinetics of dissolved macromolecules onto the interface and on how fast they unfold and acquire their new conformational state at the interface.

It is important to note that the diffusivity of the protein in the fluid will not play a role. First, the protein concentration in the fluid elements that constitute the new surface is the same as in the bulk, since the uniaxial extension will convect solution with the bulk protein concentration to the surface at the same rate as the surface area is created, so there is no need for the protein to diffuse first to the newly created surface. Second, diffusivity does not play a role as the dilatation dynamic of the interface is much faster than diffusion timescales of the protein. The ratio between the timescale of diffusion driven migration of the protein to the surface $\tau_{D}$ and the timescale of surface dilatation $\tau_{\sigma}$ describes a dimensionless Peclet number $P e=\tau_{D} / \tau_{\sigma}$. The diffusive timescale of the protein can be described following Philips and Graham [49] via 
$\tau_{D}=V \delta / D_{s} A$, where $V / A$ is the volume-to-surface area ratio, $D_{s}$ the diffusion coefficient, and $\delta$ the characteristic thickness of the diffusion layer. Setting $\delta$ equal to the filament radius $D / 2$ and assuming volume conservation of the thinning filament yields $\tau_{D}=D^{2} /\left(8 D_{s}\right)$. The timescale of the surface dilatation is taken as the inverse of the dilatation rate $\tau_{\sigma}=\dot{\varepsilon}_{\sigma}^{-1}$. Using the dilatation rate definition of eq. (5) and assuming that surface evolution is solely controlled by the thinning dynamics of the bulk solution and therefore by eq. (2) and (3), we obtain $\tau_{\sigma}=D \eta_{E, \text { app }} / \gamma$. The Peclet number for the thinning filament is then

$$
P e=\frac{\tau_{D}}{\tau_{\sigma}}=\frac{D \gamma}{8 D_{s} \eta_{E, a p p}},
$$

and is depending on the filament diameter as a critical length scale. Outgoing from a Peclet number of $P e=1$ as the critical condition at which a diffusive transport of protein molecules to the surface will overcome the creation rate of new surface, we can formulate a critical diameter of the filament below which $P e<1$ :

$$
D_{c r i t, P e}=\frac{8 D_{s} \eta_{E, a p p}}{\gamma},
$$

With $D_{s} \sim 10^{-10} \mathrm{~m}^{2} / \mathrm{s}$ for native BSA [50], a surface tension for TFE of $\gamma \sim 20.6 \mathrm{mN} / \mathrm{m}$ and an apparent extensional viscosity of $\eta_{E, a p p}=1.4 \mathrm{~Pa}$ s (taken from Fig. 4), the critical diameter calculates to $D_{c r i t, P e}=0.054 \mu \mathrm{m}$. Diffusivity is therefore not of importance for the interfacial properties of the protein solutions filaments investigated here. It is therefore solely the kinetic of adsorption to the surface that controls the protein concentration at the interface of the jet (previous studies on diffusion-controlled timescales of BSA suggest only a minor decrease of $D_{s}$ in between the native and denaturized (unfolded) state $[47,50]$, and therefore a minor decrease of $D_{\text {crit }, P e}$ for the unfolded BSA in TFE (solution \#3)).

The properties of the new surface will only be determined by the initial protein concentration at the surface and the average state of adsorption of these proteins to the surface. BSA exhibits a high degree of hydrophobicity at the interior of the folded structure. During the adsorption process to the interface of water-based solutions the protein molecules unfold (within the constraints of the intra-molecular forces of the protein structure and the denaturation state [51]), in order to expose the hydrophobic regions to the gas phase $[48,51,52]$. 
Important for the absorption kinetics in the dilating interface is the rate of configurational reorganization $r_{a d s}$ of the unfolding polymers that suddenly perceive the presence of the interface. It is the comparison of this rate $r_{a d s}$ of unfolding and adsorption onto the interface to the dilatation rate of the interface $\dot{\varepsilon}_{\sigma}$ (eq. (5)) that will determine the average conformational state of the polymers at the interface. When the adsorption rate $r_{a d s}$ is faster than the dilatation rate, the interface will contain mainly fully unfolded and adsorbed polymer chains. In case the adsorption is slower than the dilatation rate, the polymers at the interface will be mainly in a coiled and non-adsorbed state. A conservative estimation of the dilatation rate $\dot{\varepsilon}_{\sigma}$ via the bulk elongation rate (eq. (6)), using the smallest filament diameters in Fig. 6b (at which an onset of interfacial stabilization could earliest be expected) and the related extension rates $\dot{\varepsilon}$ in Fig. 4, results in $\dot{\varepsilon}_{\sigma} \geq 10^{3} \mathrm{~s}^{-1}$.

The rate of adsorption and configurational changes of proteins at newly created surfaces $r_{a d s}$ can be determined via the temporal evolution of the surface pressure of a newly created surface. However, attempts to determine the surface pressure evolution of newly created surfaces of solutions \#2 and \#3 showed a near instantaneous acquirement of the steady state interfacial tension for both solutions. This indicated adsorption and unfolding timescales within (or below) the time-span of the experiment, and therefore values for $r_{a d s}$ larger than $\mathrm{O}\left(10^{2} \mathrm{~s}^{-1}\right)$. This is in agreement with recent results on rapid ellipsometric measurements which report on unfolding timescales of concentrated protein solutions at the air/water interface on the milliseconds timescale [53]. Both solutions \#2 and \#3 have therefore adsorption timescales $r_{a d s}$ that can reach the dilatation rate of the surface $\dot{\varepsilon}_{\sigma}$ and could therefore build up a surface viscoelasticity.

In order to do a qualitative differentiation between the unfolding and adsorption kinetics of solution \#2 and \#3, we determined the behavior of dilute solutions as described in [53], for which the adsorption kinetics to the interface are sufficiently slowed down to allow a timeresolved observation of the surface pressure evolution. The surface pressure of BSA is reported to acquire a concentration-independent steady state value for protein concentrations larger than $0.001 \mathrm{wt} \%$ [54], hence experiments were conducted at a nominal concentration of $0.0033 \mathrm{wt} \%$ by injecting small amounts of solution \#2 or \#3 directly into the pure solvent bulk, without disturbing the free surface, and a subsequent monitoring of the surface pressure evolution. 
Although the similarity of the adsorption isotherms is kept when diluting the concentrated protein solutions [47], the relation between $r_{a d s}$ and the concentration is non-linear. The comparison of the adsorption kinetics between solution \#2 and \#3 outgoing from the diluted case can therefore be only qualitative and does not allow extrapolating to absolute values of $r_{a d s}$.

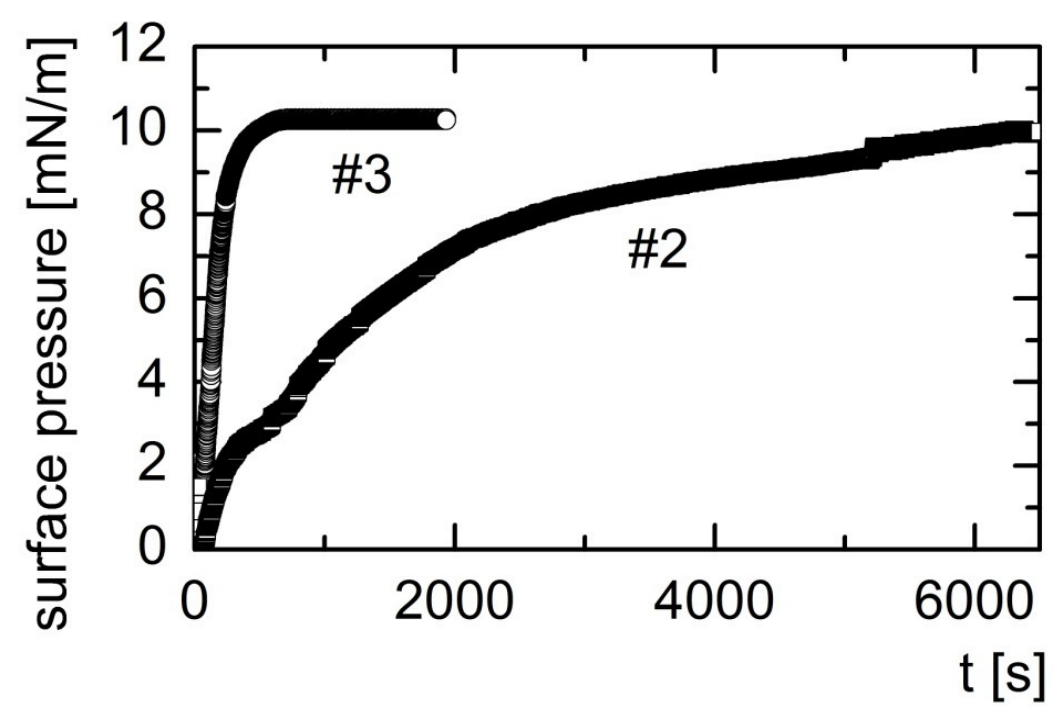

Figure 8: Relative increase of the surface pressure at the liquid/air interface over time. The samples consist of pure solvent, to which small amounts of solution \#2 and \#3 are added at $t=0$ to give nominal bulk concentrations of BSA of $c=0.0033 \%(\mathrm{w} / \mathrm{w})$.

Still, the qualitative comparison in Fig. 8 shows clearly that the dilute solution of unfolded BSA (solution \#3) shows a much faster increase in surface pressure, while the partiallyunfolded BSA (solution \#2) shows much slower dynamics. This is actually not unexpected since it is well known in literature that flexible, unfolded proteins show a quicker adsorption to the interface and a faster increase in surface pressure than globular proteins [49, 55]. A denaturation of BSA increases the exposure of the hydrophobic regions of protein in bulk solution, and therefore promotes the adsorption and arranging of the molecules at the air/solution interface [47].

Combining now the general faster adsorption kinetics of diluted solution \#3 with the observation that generally concentrated solutions show $r_{a d s}$ larger than $\mathrm{O}\left(10^{2} \mathrm{~s}^{-1}\right)$, indicates that the adsorption rate of solution \#3 will reach the order of the critical dilatation rate of the 
electrospinning process $\dot{\varepsilon}_{d} \geq 10^{3} \mathrm{~s}^{-1}$, and therefore has a higher density of unfolded and adsorbed protein molecules at the newly created surface than solution \#2.

It should be noted that for the investigation of diluted protein solutions as in Fig. 8, the diffusivity of the protein molecules cannot be neglected (as for the concentrated solutions), since the transport of the proteins from the injection point to the surface will also affect the adsorption rate. On the basis of classical diffusion theory (Stokes-Einstein), the fully-unfolded protein (solution \#3) should have a lower diffusivity than this of the partially-unfolded one (solution \#2), as the frictional coefficient of the former would be greater. However, the results in Fig. 8 show a much faster adsorption kinetics of the fully-unfolded BSA onto the interface, despite this prediction of lower diffusivity, which implies an energy barrier controlled adsorption rather than diffusion controlled adsorption for the investigated BSA solutions. This dominance of an energy barrier controlled adsorption has already been reported for BSA in the globular state [56], and it is apparently also the reason for the observed large difference in the adsorption kinetics in the present investigation of the partially- and fully- unfolded proteins of solutions \#2 and \#3, respectively. Nevertheless, Damodaran et al. [57] studied the adsorption kinetics of several conformational intermediates of BSA and suggested that neither diffusion nor energy barrier alone governor the adsorption kinetics of protein, but also its conformational state in the solution (their results are in accordance with our results (Fig. 8) - the rate of increase of surface pressure at the air/solution interface of dilute protein solution is greater as the protein is more unfolded).

\subsection{Discussion}

The qualitative evaluation of the interfacial experiments have demonstrated two things: the adsorption kinetics of protein molecules can be fast enough to form a surface layer even with dilatation rates as experienced in the electrospinning process; and this interfacial layer has a high enough interfacial viscosity to dominate the axial stress distribution below a critical filament radius that is relevant to the electrospinning process. Furthermore, the kinetic and rheological experiments show an order of magnitude difference in the adsorption rate and the interfacial viscoelasticity between the partially unfolded state of the proteins in solution \#2 and the fully unfolded state in solution \#3. The denaturation of the proteins in solution \#3 is therefore a requirement in order to achieve interfacial properties that sufficiently contribute to the stabilization of the jet during the electrospinning of protein solution. 
It should be noted that the static interfacial experiments do not directly mimic the unique conditions of the electrospinning process. Although qualitatively showing that interfacial viscoelasticity will have a significant influence on the stabilization of the filament, the electrical field and mass transfer due to the evaporating solvent will both have an additional influence on the interfacial properties.. The additional strong extensional stretching and the electric field acting on the charged molecules will likely result in an active stretching and unfolding of the proteins, increasing the exposure of the hydrophobic areas to the air interface. Therefore, an acceleration of the kinetics of the protein adsorption at the surface is expected. Both of these effects remains detail of further investigation

\section{Summary}

In this study, it was demonstrated that a protein solution with no significant bulk elastic component can be electrospun into continuous fibers. The essential role of jet stabilization is hypothesized to be fulfilled by the viscoelastic response of the eventually solidifying surface layer. The kinetics of the skin formation and the surface mechanical behavior are affected by the protein conformation in the solutions, in addition to the inherent solvent volatility. A more open (unfolded) structure for the chain is assumed to promote the adsorption kinetics onto the interface and to cause higher interfacial moduli. The interfacial viscoelasticity is shown to be sufficient to deliver axial forces that overcome the forces in the bulk liquid below a filament diameter on the order of $\sim 80 \mu \mathrm{m}$. Taken together, these effects can explain the stabilization of the jet in the spinnable, denaturized protein solution (solution \#3), in contrast to the unspinnable and partially-spinnable solutions (solutions \#1 and \#2, respectively). Furthermore, these results suggest the important role of viscoelastic skin formation during electrospinning, even for polymer systems that exhibit significant measurable bulk elasticity.

\section{Acknowledgements}

CC is acknowledging financial support from the ERC starting grant 203043 NANOFIB and EZ acknowledging the RBNI at the Technion for their financial support. 


\section{References}

1. Reneker DH, Yarin AL, Zussman E, Xu H. Advances in Applied Mechanics 2007;41:43-195.

2. Hohman MM, Shin M, Rutledge G, Brenner MP. Physics of Fluids 2001;13:2201-20.

3. Hohman MM, Shin M, Rutledge G, Brenner MP. Physics of Fluids 2001;13:2221-36.

4. Deitzel JM, Kleinmeyer J, Harris D, Beck Tan NC. Polymer 2001;42:261-72.

5. Theron SA, Zussman E, Yarin AL. Polymer 2004;45:2017-30.

6. Shin YM, Hohman MM, Brenner MP, Rutledge G. Applied Physics Letters 2001;78:1149-51.

7. McKinley GH, Sridhar T. Annual Review of Fluid Mechanics 2002;34:375-415.

8. Helgeson ME, Grammatikos KN, Deitzel JM, Wagner NJ. Polymer 2008;49:2924-36.

9. Yu JH, Fridrikh SV, Rutledge GC. Polymer 2006;47:4789-97.

10. Shenoy SL, Bates WD, Frisch HL, Wnek GE. Polymer 2005;46:3372-84.

11. Bhattacharjee PK, Oberhauser JP, McKinley GH, Leal LG, Sridhar T. Macromolecules 2002;35:10131-48.

12. Eggers J. Reviews of Modern Physics 1997;69:865-929.

13. Eggers J, Villermaux E. Reports on Progress in Physics 2008;71:036601.

14. Larson RG. Rheologica Acta 1992;31:213-63.

15. Clasen C, Plog JP, Kulicke W-M, Owens M, Macosko C, Scriven LE, Verani M, McKinley GH. Journal of Rheology 2006;50:849-81.

16. Clasen C, Eggers J, Fontelos MA, Li J, McKinley GH. Journal of Fluid Mechanics 2006;556:283-308.

17. Kwon IK, Matsuda T. Biomacromolecules 2005;6:2096-105.

18. Klossner RR, Queen HA, Coughlin AJ, Krause WE. Biomacromolecules 2008;9:2947-53.

19. Varesano A, Aluigi A, Vineis C, Tonin C. Journal of Polymer Science: Part B: Polymer Physics 2008;46:1193-201.

20. Dror Y, Ziv T, Makarov V, Wolf H, Admon A, Zussman E. Biomacromolecules 2008;9:2749-54.

21. Theron SA, Zussman E, Yarin AL. Nanotechnology 2001;12:384-90.

22. Yarin AL, Zussman E, Theron A, Rahimi S, Sobe Z, Hasan D. Journal of Rheology 2004;48:101-16. 
23. Anna SL, Rogers C, McKinley GH. Journal of Non-Newtonian Fluid Mechanics 1999;87:307-35.

24. Anna SL, McKinley GH, Nguyen DA, Sridhar T. Journal of Rheology 2001;45:83-114.

25. Vandebril S, Vermant J, Moldenaers P, Franck A, Fuller GG. 5th Annual European Rheology Conference (AERC), Cardiff, UK, 2009.

26. Vandebril S, Franck A, Fuller GG, Moldenaers P, Vermant J. Rheologica Acta 2009; In Press.

27. Arinstein A, Zussman E. Physical Review E 2007;76:056303 1-7.

28. Ikeda S, Nishinari K. Biomacromolecules 2000;1:757-63.

29. Kundu A, Kishore N. Biophysical Chemistry 2004;109:427-42.

30. Banerjee T, Kishore N. Biopolymers 2005;78:78-86.

31. Carrotta R, Manno M, Giordano FM, Longo A, Portale G, Martorana V, Biagio PLS. Physical Chemistry Chemical Physics 2009;11:4007-18.

32. Kowalewski TA, Barral S, Kowalczyk T. IUTAM Symposium on Modelling Nanomaterials and Nanosystems, Aalborg, Denmark, 2008.

33. Plog JP, Kulicke W-M, Clasen C. Applied Rheology 2005;15:28-37.

34. Yesilata B, Clasen C, McKinley GH. Journal of Non-Newtonian Fluid Mechanics 2006;133:73-90.

35. Rodd LE, Scott TP, Cooper-White JJ, McKinley GH. Applied Rheology 2005;15:12-27.

36. Anna SL, McKinley GH. Journal of Rheology 2001;45:115-38.

37. McKinley GH, Tripathi A. Journal of Rheology 2000;44:653-70.

38. Entov VM, Hinch EJ. Journal of Non-Newtonian Fluid Mechanics 1997;72:31-53.

39. Edwards DA, Brenner H, Wasan DT. Interfacial transport processes and rheology Boston:Butterworth-Heinemann; 1991).

40. Reynaert S, Brooks CF, Moldenaers P, Vermant J, Fuller GG. Journal of Rheology 2008;52:261-85.

41. Ariola FS, Krishnan A, Vogler EA. Biomaterials 2006;27:3404-12.

42. Bos MA, van Vliet T. Advances in Colloid and Interface Science 2001;91:437-71.

43. Wang ZB, Narsimhan G. Langmuir 2005;21:4482-9.

44. Freer EM, Yim KS, Fuller GG, Radke CJ. Journal of Physical Chemistry B 2004;108:383544. 
45. Kojic N, Bico J, Clasen C, McKinley GH. Journal of Experimental Biology 2006;209:435562.

46. Ewoldt RH, Clasen C, Hosoi AE, McKinley GH. Soft Matter 2007;3:634-43.

47. McClellan SJ, Franses EI. Colloids and Surfaces B: Biointerfaces 2003;28:63-75.

48. Lu JR, Su TJ, Penfold J. Langmuir 1999;15:6975-83.

49. Graham DE, Phillips MC. Journal of Colloid and Interface Science 1979;70:403-14.

50. Cho D, Narsimhan G, Franses EI. Journal of Colloid and Interface Science 1997;191:312-25.

51. Graham DE, Phillips MC. Journal of Colloid and Interface Science 1979;70:427-39.

52. Wang J, Buck SM, Chen Z. Analyst 2003;128:773-8.

53. Wierenga PA, Egmond MR, Voragen AGJ, de Jongh HH. Journal of Colloid and Interface Science 2006;299:850-7.

54. Graham DE, Phillips MC. Journal of Colloid and Interface Science 1979;70:415-26.

55. Mitchell J, Irons L, Palmer GJ. Biochimica Et Biophysica Acta 1970;200:138-\&.

56. Damodaran S, Song KB. ACS Symposium Series 1991;454:104-21.

57. Damodaran S, Song KB. Biochimica Et Biophysica Acta 1988;954:253-64. 